\title{
Polarity-Controlled GaN/AlN Nucleation Layers for Selective-Area Growth of GaN Nanowire Arrays on Si (111) Substrates by Molecular Beam Epitaxy
}

Matt D. Brubaker, Shannon M. Duff, Todd E. Harvey, Paul T. Blanchard, Alexana Roshko, Aric W. Sanders, Norman A. Sanford, and Kris A. Bertness

Supplemental Figures:

(a)

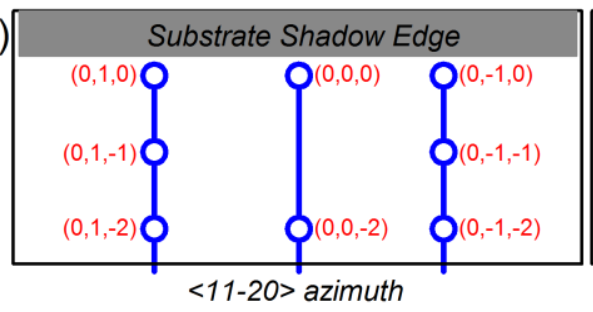

(b)

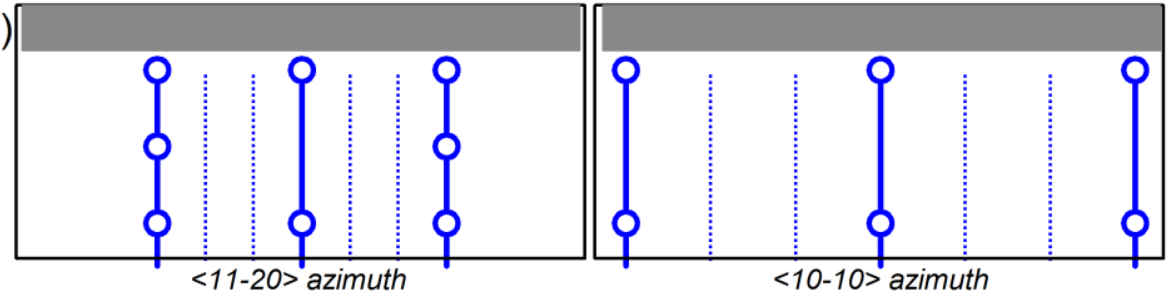

(c)

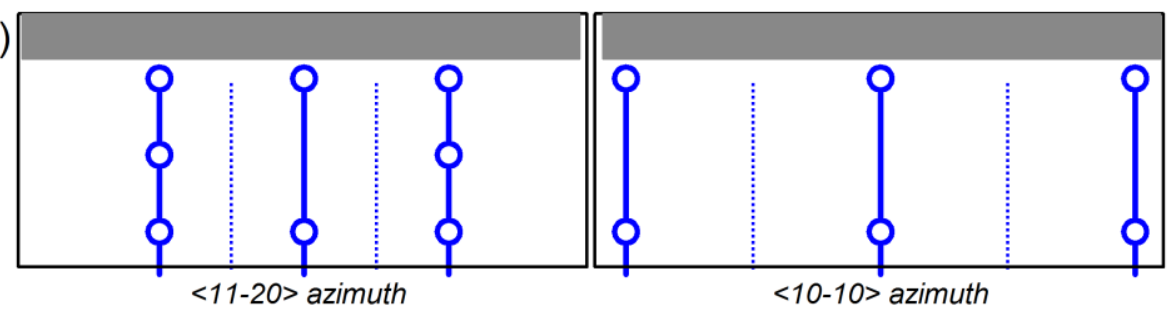

Figure S1. Calculated RHEED patterns showing spot and streak locations for (a) 1x1, (b) 3x3, and (c) $2 \times 2$ reconstructions. The open circles are spot locations (associated with 3D transmission patterns), the solid lines are streaks (associated with 2D planar diffraction), and the dotted lines are fractional order streaks resulting from surface reconstructed supercells. Diffraction spots are indexed in red text in (a), assuming an N-polar orientation. 

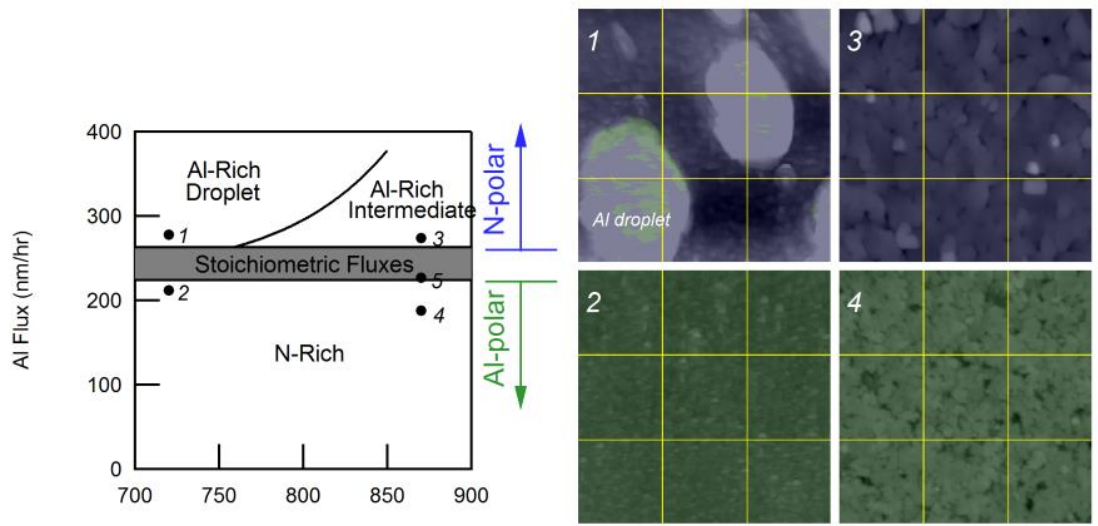

Growth Temperature $\left({ }^{\circ} \mathrm{C}\right)$

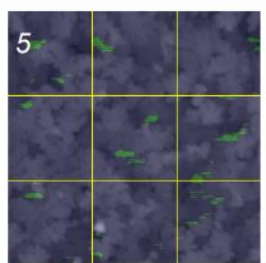

Figure S2. AlN growth diagram and corresponding PFM phase/topography composite images. $\mathrm{N}$ - or Al-polarity is indicated by the blue or green overlays, respectively, on a $1 \mu \mathrm{m}$ grid. Al droplets are observed for low-temperature, metal-rich growth conditions (1), while mixedpolarity films are observed at near-stoichiometric fluxes (5). Otherwise, the AlN films adopt Al(N-) polarity for N- (Al-) rich growth conditions.
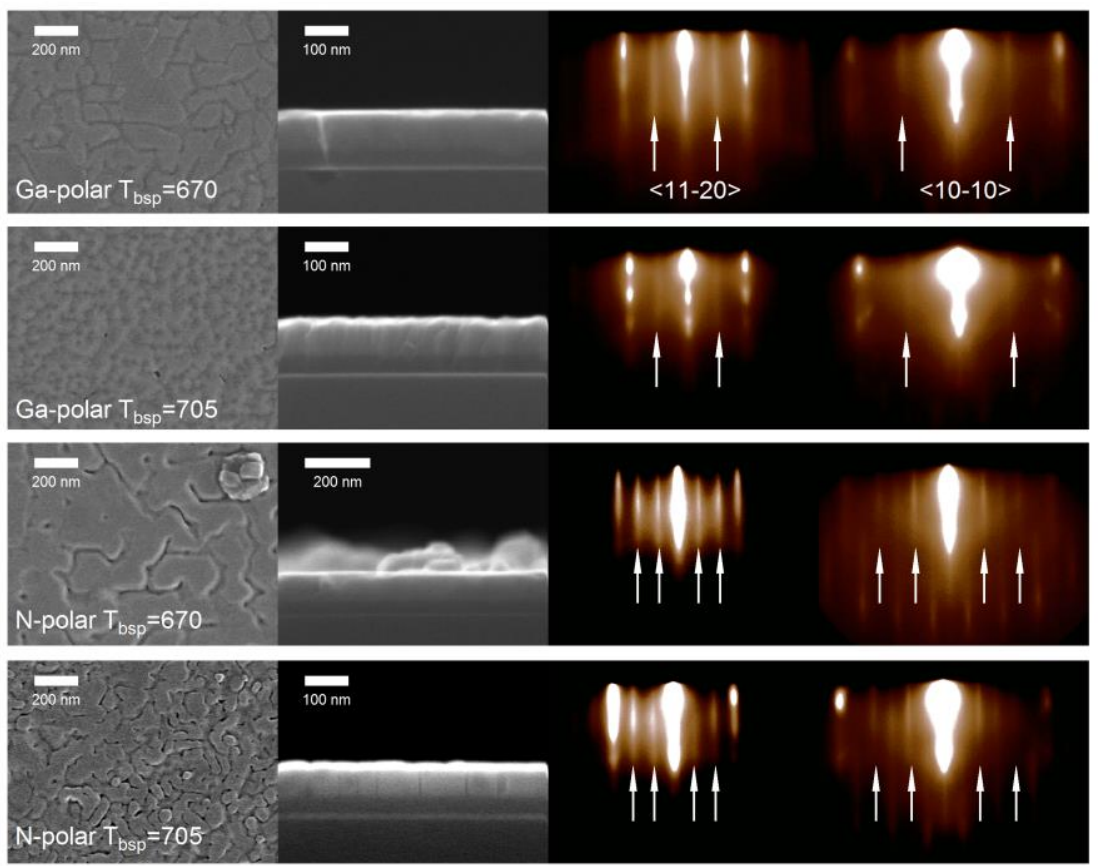

Figure S3. FESEM images and corresponding post-cooldown RHEED images of N- and Gapolar GaN nucleation layers, used for partial optimization of surface morphology. Based on these samples a growth temperature of $670^{\circ} \mathrm{C}\left(705^{\circ} \mathrm{C}\right)$ was chosen for $\mathrm{Ga}-(\mathrm{N}-)$ polar $\mathrm{GaN}$ nucleation layers. 

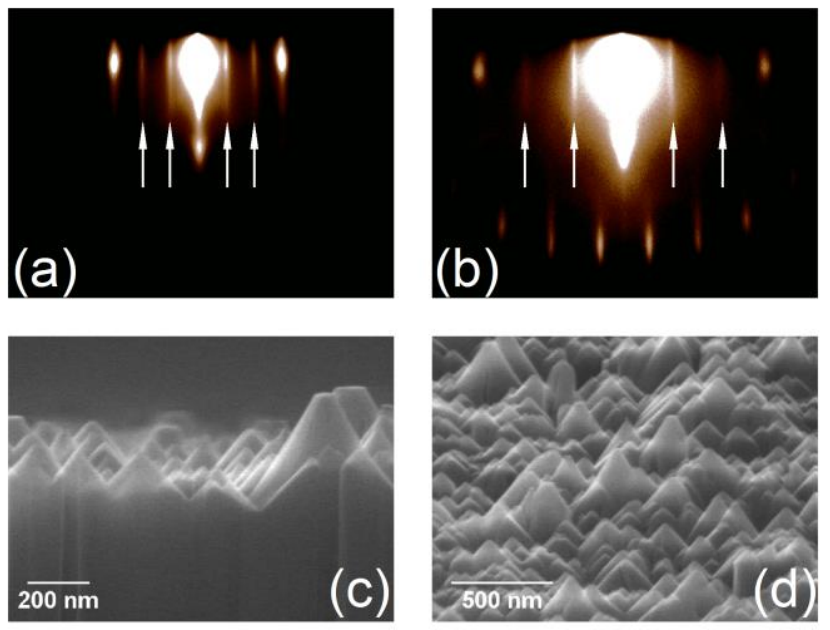

Figure S4. Polarity measurements for 1 um thick N-polar GaN nucleation layer, showing postcooldown RHEED patterns in (a) $<11-20>$ and (b) $<10-10>$ azimuths and (c,d) polaritysensitive-etch tests. The $3 \times 3$ surface reconstruction and pyramidal etch features in $(c, d)$ correlate well with the expected results outlined in Table 1.
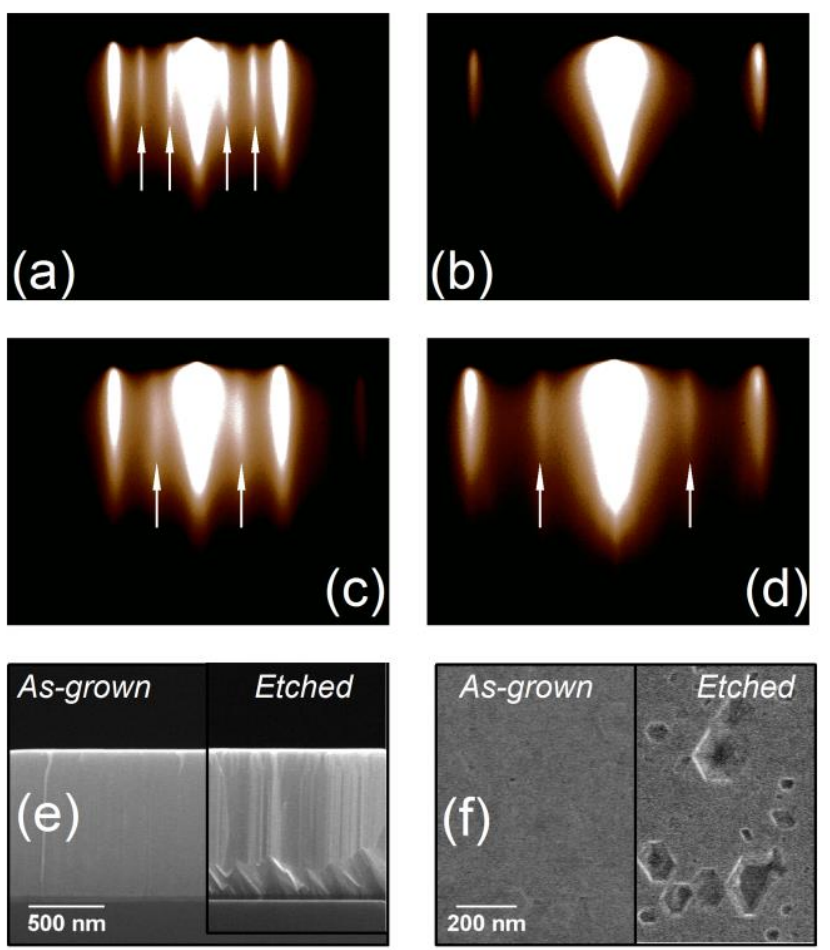

Figure S5. Polarity measurements for 1 um thick Ga-polar GaN nucleation layer, showing postcooldown RHEED patterns in $(\mathrm{a}, \mathrm{c})<11-20>$ and $(\mathrm{b}, \mathrm{d})<10-10>$ azimuths and (e,f) polaritysensitive-etch tests. A polarity-ambiguous $3 \times 1$ pattern is observed $(a, b)$ when excess surface $\mathrm{Ga}$ is present, reverting to the Ga-polar $2 \times 2$ pattern $(\mathrm{c}, \mathrm{d})$ upon additional exposure to active nitrogen flux. The $2 \times 2$ surface reconstruction in $(c, d)$ and hexagonal etch pits in $(e, f)$ correlate well with the expected results outlined in Table 1. 